\title{
Predictive and Characterized Pulsar Evolution From its Optical Pulsation
}

\author{
Rizchel Masong ${ }^{1,2^{*}}$ \\ ${ }^{1}$ De La Salle University Manila, College of Science, Physics Department, 2401, Taft avenue, Manila, \\ Philippines. \\ ${ }^{2}$ De La Salle Araneta University, College of Arts and Sciences, Science Department, Victoneta \\ Avenue, Malabon, Philippines
}

\begin{abstract}
The measured and predicted variables of pulsar PSR B1937+21 were explored from the energetics obtained from the previous observation made. The target parameters such as the brightness, luminosities, and optical efficiency. A series of observations were analysed to determine the individual values of the parameters chosen. Within the extended area of optical pulsation, the values were plotted to determine the relation of it to synchrotron emission. The results were presented graphically to see whether PSR B1937+21 will remain consistent with the expected pulsar model. The researcher suggests that simulation and correlation of previous observation made together with a new one to explore the cause of optical pulsation from mass transfer and spectral evolution.
\end{abstract}

\section{Introduction}

Pulsar is known as is a highly magnetized rotating neutron star that emits beams of electromagnetic radiation out of its magnetic poles and originally know to be observed under radio waves. However, there is a surprising event that happened in PSR J1023+0038. There was a state change that occurred in PSR J1023. There was a disappearance of radio pulsations and a development of accretion disk (Stappers, Patruno, Tendulkar, 2014) in a simultaneous observation and study. Further strengthening the idea that most of the optical and X-ray luminosity comes from an accretion process and not from an intrabinary shock or from the spin-down luminosity of a rotation-powered pulsar (Patruno, 2014). Since, we saw that radio pulsation disappears as accretion disk develops. What type of pulsations can we now detect? X-ray/optical correlation found in NS LMXBs with the addition of J1023. The correlation exists between the reprocessed light and the X-ray emission coming from the accretion process (Patruno, et al. 2014). A pulsar is also observable under the optical, Xray, and UV regime (Ambrosino, et al. 2018) on similar pulsar PSR J1023. Optical pulsed emission could be due to synchrotron emission by relativistic electrons in the magnetosphere of a rotationpowered pulsar. This also demonstrates that the magnetosphere of old, weakly magnetic and

\footnotetext{
* Corresponding author: rizchel_masong@dlsu.edu.ph
} 
quickly spinning neutron stars can give rise to such signals when surrounded by an accretion disk

PSR B1937+21 (PSR J1939+2134) on the other hand, is a pulsar located in the constellation Vulpecula and was the first millisecond (MS) pulsar to be discovered (Backer et al. 1982), it has a period of $1.56 \mathrm{~ms}$, and was the fastest-spinning known pulsar. The profile of PSR B1937+21 consists of a main pulse, which has at least two components spaced close to each other, and an interpulse and the separation angle. $\Delta \varphi_{M P-I P}=174^{\circ}$ remains constant for a wide frequency range, from 0.32 to $2.38 \mathrm{GHz}$ (Stinebring \& Cordes 1983). The constant $\Delta \varphi_{M P-I P}$ could be perceived as an indication that the emission originates from two different magnetic poles. This view is further supported by the lack of low-level emission for most of the period, at least with the current observations (e.g., Camilo 1995). It came as a surprise to find qualitatively similar behavior from PSR B1937+21 that shows frequent, very strong radio pulses extending to hundreds of times the mean pulse intensity. the first (and still fastest known) millisecond pulsar.

The Radhakrishnan \& Cooke (1969) explanation of the Monotonic sweep of linear polarization across the Vela pulsar's average pulse profile in terms of this polar cap model provided the cornerstone upon which most subsequent work has been based (Sallmen \& Backer 1995; Cognard et al. 1996). When a pulsar rotates fast enough the separation between tangents to the outermost field lines which connect to the interstellar medium spreads in proportion to $P^{-0.5}$ for a simple dipole geometry when measured at the same altitude. If pulsar radiation fills a fixed fraction of this 'open' field zone, then we could expect a similar scaling of observed pulse width, measured in angle not time, with period. A dependency of emission altitude on period can, of course, alter this relation. Usual analysis and studies where made in relating width-period relation since the early days of pulsar discovery. The technique demonstrated the dependence of pulsar property in objects within the same line of sight, dipole axis, and spin axis geometries were observed and compared (Lyne \& Manchester 1988). What arises from these studies is that the central component of the beam provided by pulsar rotation has a steep spectrum. And also displays sign reversal of circular polarization.

PSR B1937+21 (also referred to as PSR J1939+2134) was the first MSP discovered (Backer et al. 1982) and for 25 years, the fastest spinning pulsar known ( $\mathrm{P}=1.55 \mathrm{~ms})$. With a spindown luminosity of $\dot{E}=1.1 \times 10^{36} \mathrm{erg} \mathrm{s}^{-1}$, it is the second most energetic MSP detected. This isolated pulsar lies in the Galactic plane at a distance of $\sim 3.5 \mathrm{kpc}$. At X-ray energies, PSR B1937+21 has been detected as a pulsed source with ASCA (Takahashi et al. 2001), BeppoSAX (Nicastro et al. 2004), RXTE (Guillemot et al. 2012), Chandra, and XMMNewton (Ng et al. 2014). In hard X-rays, the pulsar has so far only been detected up to $\approx 13$ $\mathrm{keV}$ with RXTE (Guillemot et al. 2012). Like PSR B1821-24, this MSP shows sharp pulsations with a small duty cycle and nearly $100 \%$ pulsed fraction. In this paper, we present an analysis of the optical properties of PSR B1937+21 in terms of the observation made by NuSTAR, Chandra, and XMM-Newton data, and archived in ATNF Pulsar Catalogue made by CSIRO (Manchester, R. N., Hobbs, G.B., Teoh, A. \& Hobbs, 2005)

\section{Observations and Data Analysis}

The observations used were made using the Large European Array for Pulsars (LEAP); a tied-array telescope comprised of various combinations of five European 100-m class radio telescopes: the Effelsberg Telescope, the Lovell Telescope, the Nancay Radio Telescope 
(NRT), the Westerbork Synthesis Radio Telescope (WSRT), and the Sardinia Radio Telescope (SRT). Observations with the participating telescopes are made simultaneously and are combined coherently into a tied-array beam, forming a virtual 195-m dish when using all five telescopes (McKee et. al, 2018).

Table 1. Summary of the LEAP observation of PSR B1937+21.

\begin{tabular}{|c|c|c|c|c|c|c|c|c|c|c|}
\hline Date & MJD & Telescopes & $f_{c}(\mathrm{MHz})$ & BW $(\mathrm{MHz})$ & $T_{\mathrm{obs}}(\mathrm{sec})$ & $\mathrm{S} / \mathrm{N}_{\text {profile }}$ & Coherency & $N_{\mathrm{MGP}}$ & $N_{\text {IGP }}$ & $N_{\mathrm{GP}}$ \\
\hline $2012-09-23(1)$ & 56193 & EJW & 1412 & 64 & 1240 & 719 & $80 \%$ & 125 & 77 & 202 \\
\hline 2012-09-23 (2) & 56193 & EJW & 1412 & 64 & 1020 & 808 & $86 \%$ & 124 & 74 & 198 \\
\hline 2013-07-27 & 56500 & EJW & 1404 & 112 & 1800 & 525 & $63 \%$ & 138 & 106 & 244 \\
\hline 2013-08-25 & 56529 & EW & 1364 & 64 & 2700 & 1065 & $98 \%$ & 278 & 154 & 432 \\
\hline 2014-05-22 & 56799 & ESW & 1396 & 128 & 2760 & 904 & $86 \%$ & 168 & 83 & 251 \\
\hline 2014-07-07 & 56845 & EW & 1420 & 80 & 2770 & 435 & $88 \%$ & 172 & 84 & 256 \\
\hline 2014-07-27 & 56865 & EW & 1380 & 96 & 2760 & 492 & $97 \%$ & 31 & 22 & 53 \\
\hline 2014-08-24 & 56893 & EJNW & 1396 & 128 & 2490 & 852 & $92 \%$ & 155 & 75 & 230 \\
\hline 2014-10-15 & 56945 & EJNW & 1380 & 64 & 1470 & 587 & $57 \%$ & 139 & 76 & 215 \\
\hline 2015-02-25 & 57078 & EJNW & 1396 & 128 & 2120 & 715 & $76 \%$ & 145 & 94 & 239 \\
\hline 2015-03-26 & 57107 & EJNSW & 1396 & 128 & 2510 & 1232 & $82 \%$ & 171 & 87 & 258 \\
\hline 2015-04-17 & 57129 & EJNSW & 1396 & 128 & 2510 & 1202 & $80 \%$ & 137 & 52 & 189 \\
\hline 2015-06-20 & 57193 & EJW & 1396 & 128 & 2230 & 598 & $100 \%$ & 112 & 63 & 175 \\
\hline 2015-07-19 & 57222 & EJS & 1412 & 96 & 2790 & 458 & $67 \%$ & 112 & 92 & 204 \\
\hline 2015-09-18 & 57283 & EJN & 1404 & 112 & 2520 & 429 & $78 \%$ & 90 & 32 & 122 \\
\hline 2015-10-10 & 57305 & EJ & 1412 & 96 & 2790 & 543 & $86 \%$ & 112 & 52 & 164 \\
\hline 2015-11-07 & 57333 & EJS & 1396 & 128 & 2790 & 729 & $99 \%$ & 81 & 55 & 136 \\
\hline 2015-12-12 & 57368 & EJNS & 1396 & 128 & 2520 & 755 & $75 \%$ & 138 & 55 & 193 \\
\hline 2016-01-09 & 57396 & EJNS & 1396 & 128 & 2520 & 1122 & $83 \%$ & 154 & 99 & 253 \\
\hline 2016-02-05 & 57423 & EJNS & 1404 & 112 & 2390 & 629 & $75 \%$ & 97 & 49 & 146 \\
\hline 2016-04-07 & 57485 & EJNS & 1388 & 80 & 2380 & 924 & $78 \%$ & 110 & 48 & 158 \\
\hline Mean & - & - & - & - & 2337 & 709 & $82 \%$ & 133 & 70 & 203 \\
\hline Total & - & - & - & - & 49080 & - & - & 2789 & 1476 & 4265 \\
\hline
\end{tabular}

Monthly observations have been made in LEAP mode since February 2012, and is presented in Table 1. It consists of the telescope used in the observation proper, the frequency and bandwidth, and the duration of the observations made. There is a difference in bandwidth and either caused by instrumental setup or due to data loss. Also, the ephemeris of PSR B19387+21 and derived parameters. Obtained from observations with the Effelsberg 100-m telescope and Arecibo archival data. Figures in parentheses represent uncertainties $(2 \sigma)$ in the last digits quoted.

Table 2. Summary of the LEAP observation of PSR B1937+21.

\begin{tabular}{|c|c|}
\hline Parameter & Value \\
\hline Right Ascension (J2000) & $19^{\mathrm{h}} 39^{\mathrm{m}} 38^{\mathrm{s}} .5600084(8)$ \\
\hline Declination (J2000) & $21^{\circ} 34^{\prime} 59^{\prime \prime} .13548(14)$ \\
\hline Frequency $(f, \mathrm{~Hz})$ & $641.928246349481(9)$ \\
\hline Freq. derivative $\left(\dot{f}, \mathrm{~Hz} \mathrm{~s}^{-1}\right)$ & $-4.330999(2) \times 10^{-14}$ \\
\hline Freq. 2 nd deriv. $\left(\ddot{f}, \mathrm{~Hz} \mathrm{~s}^{-2}\right)$ & $1.558(8) \times 10^{-26}$ \\
\hline $\mathrm{DM}^{\mathrm{a}}\left(\mathrm{cm}^{-3} \mathrm{pc}\right)$ & $71.03998(6)$ \\
\hline Epoch of ephemeris (MJD) & 52328.0 \\
\hline Range of validity (MJD) & $45986-52764$ \\
\hline RA proper motion ( $\operatorname{mas} \mathrm{yr}^{-1}$ ) & $-0.128(8)$ \\
\hline Dec proper motion $\left(\operatorname{mas} \mathrm{yr}^{-1}\right)$ & $-0.486(12)$ \\
\hline Gal latitude $(l)$ & 57.5215 \\
\hline Gal longitude $(b)$ & -0.2697 \\
\hline Parallax $(\pi$, mas $)$ & $<0.28$ \\
\hline Distance $^{\mathrm{b}}(d-$ from DM, kpc) & 3.6 \\
\hline Surface magnetic field $\left(B_{S}, \mathrm{G}\right)$ & $4.1 \times 10^{8}$ \\
\hline Light-cylinder $B\left(B_{L}, \mathrm{G}\right)$ & $1.0 \times 10^{6}$ \\
\hline Spin-down energy loss $\left(\dot{E}, \mathrm{erg} \mathrm{s}^{-1}\right)$ & $1.1 \times 10^{36}$ \\
\hline$\dot{E} / 4 \pi d^{2}\left(\operatorname{erg~} \mathrm{cm}^{-2} \mathrm{~s}^{-1}\right)$ & $7.1 \times 10^{-10}$ \\
\hline
\end{tabular}


Exploring the temporal analysis made (Cusamano, et. al, 2003), showed that the folded light curve of the MECS data together with the radio one obtained with the 100- $\mathrm{m}$ Effelsberg radio-telescope of the LEAP network. On the other hand, Figure 2 shows the X-ray pulse profile in 3 energy ranges. Non-detection of the interpulse in the ASCA data is explained by their lower statistical significance (227 total photons in $1.7-6.5 \mathrm{keV}$ ).

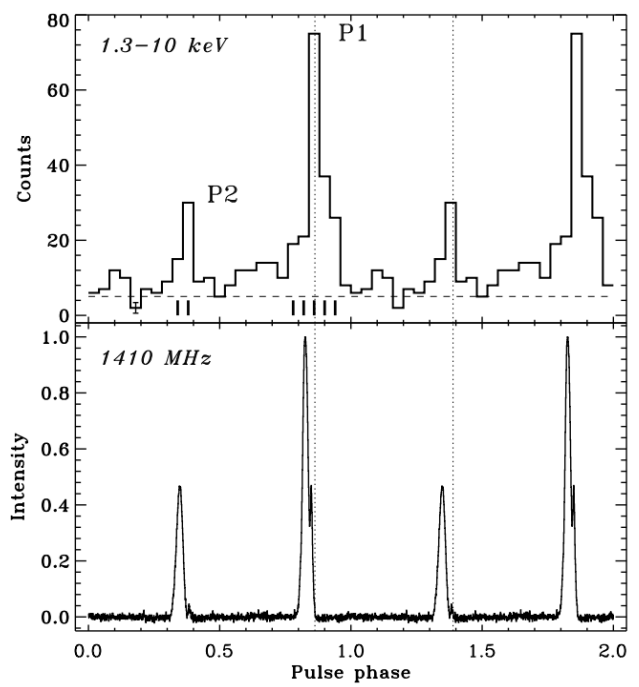

Figure 1. Radio pulse profile and the $1.3-10 \mathrm{keV}$ BeppoSAX X-ray profile phase aligned with the radio giant pulses.

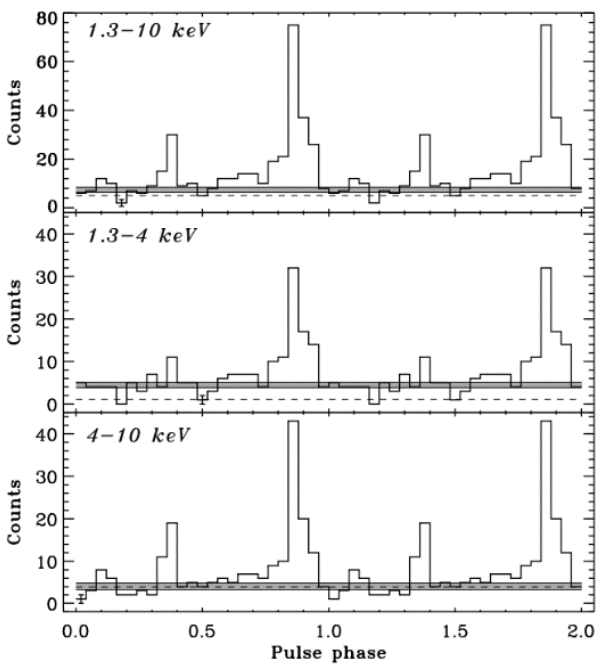

Figure 2. The X-ray pulse profile in the full MECS X-ray band $1.3-10 \mathrm{keV}$ and two subbands.

From the observations made. Exploring the optical property of pulsar in terms of its brightness, spin down luminosities, and optical efficiency made it possible. For giant pulses of pulsars, the energy density is comparable with that of oscillation in the inner gap. It means that we obtain radiation at the giant pulsar instants directly from the gap by-passing the magnetosphere plasma. This explains that pulsars as an emitter has a main characteristic which known as the brightness temperature and spectrum as well as polarization that can be considered as grounds for analysis (Kontorovich, 2015). The brightness $b$ is defined by equation (1).

$$
b=\frac{L}{4 \pi d^{2}}
$$

Based on this, we can view the main emission properties of millisecond pulsar which show non-thermal emission only, in order to account for the contribution to the flux by the field source listed by Becker et al. (2003). We can also explore the spin down rate based on the given logarithm of luminosity and logarithm of spin down rate.

As a pulsar emits radiation, the energy is extracted from its rotation. By observing the luminosity of a pulsar's nebula, we can estimate a rate at which pulsar is slowing down. This is known as the spin-down luminosity which is defined by the equation (2) which is the ratio from the $-\dot{E}$. 


$$
-\dot{E}=\frac{4 \pi^{2} I \dot{P}}{P^{3}}
$$

Optical efficiency on the other hand is the ratio of the luminosity and it's spin down power luminosity. Since the luminosity can be obtain from the solution of equation 2 , we can obtain its ratio with the spin-down luminosity. The value can be achieved by the equation 3 .

$$
\eta_{\text {opt }}=\frac{L_{x}}{\dot{E}_{s d}}
$$

Based on the mentioned parameters together with the observations made. Exploration about the pulsar property will be interpreted.

\section{Results}

Since the energetics was obtained from the previous observations made for PSR B1937+21, luminosity can be easily measure and interpret. We are expecting a value of brightness that is equivalent to $3.6 \times 10^{6} \mathrm{Wm}^{-2}$. And by plotting the optical luminosities $\log L_{x}$, and $\log \dot{E}$ showed in figure 3 shows the main emission properties of millisecond pulsars and demonstrates non-thermal emission. And in order to account for the contribution to the flux by the field source listed by Becker et al. (2003). We have $\log \dot{E}$ to be equivalent to $36.0 \mathrm{erg} / \mathrm{s}$. While $\log L_{x}$ to be equivalent to $31.57 \mathrm{erg} / \mathrm{s}$ and displays that pulsar PSR B1937+21 which exhibits a property of being a dipole and has a maximum luminosity for synchrotron emission.



Figure 3. Plot of logarithm of spin down luminosity and logarithm of optical luminosity with pulsars in both radio and optical regime. 


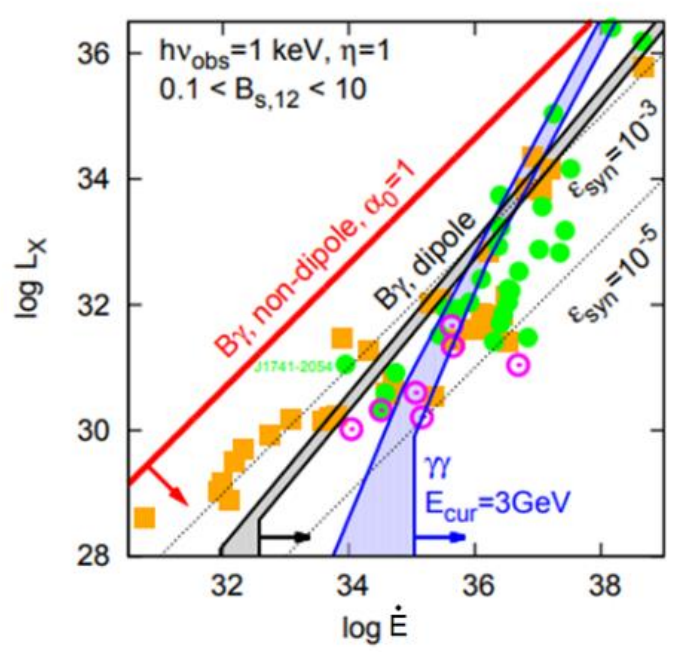

Figure 4. Maximum luminosities of synchrotron radiation in $1 \mathrm{keV}$ (upper) and $1 \mathrm{eV}$ (lower) for two-photon collision case $(\gamma \gamma$, blue), magnetic pair creation cases (B $\gamma)$ with dipole field (black) and non-dipole fields (red) as a function of the spin-down luminosity $\mathrm{L}_{\mathrm{sd}}$.

Comparing the plot of Figure 3 to Figure 4 we can see that PSR B1937+21. We can observe that pulsar PSR B1937+21 exhibits a property of being a dipole and has a maximum luminosity for synchrotron emission which confirms the pulsar model. This confirms that PSR B1937+21 has a projected optical pulsation in terms of synchrotron emission obtained from the optical luminosity and spin down luminosity. We also consider the range of the surface magnetic field $0.1<\mathrm{Bs}, 12<10 \mathrm{G}$. Its dependence of the luminosity on the magnetic field is described by $L_{s y n} \propto B-1 / 4$ s. (Kisaka and Tanaka, 2017). Exploring further the spin down luminosity and comparing it with the sun and crab pulsar. We can see that the pulsations/pulse luminosity is close by a factor of $10^{2} \mathrm{~L} \odot$ which can be seen in Table 3. From this we can agree that

Table 3. Comparison of the PSR B1937+21 and Crab Pulsar.

\begin{tabular}{ccccc}
\hline Pulsar & Period & $\begin{array}{c}\text { Moment of } \\
\text { Inertia (I) }\end{array}$ & $\dot{P}$ & $\begin{array}{c}\text { Spin Down } \\
\text { Luminosity }\end{array}$ \\
\hline PSR B1937+21 & $0.00156 \mathrm{~s}$ & $10^{45} \mathrm{~g} / \mathrm{cm}^{3}$ & $10^{-19}$ & $\begin{array}{c}10^{36} \mathrm{ergs} / \mathrm{s} \text { or } \\
3.82 \times 10^{3} \mathrm{~L} \odot\end{array}$ \\
\hline Crab Pulsar & $0.033 \mathrm{~s}$ & $10^{45} \mathrm{~g} / \mathrm{cm}^{3}$ & $10^{-12.4}$ & $\begin{array}{c}4 \times 10^{38} \mathrm{ergs} / \mathrm{s} \\
\text { or } 10^{5} \mathrm{~L} \odot\end{array}$ \\
\hline
\end{tabular}


The spin down luminosity of PSR B1937+21 is relatively closer to the value of Crab pulsar by a factor of $10^{2} \mathrm{~L} \odot$. Further comparison of apparent brightness is advisable to check the interpretation of differences in luminosity. The luminosity observed by PSR B1937+21 shows it compatibility with synchrotron emission of a standard pulsar model. Lastly, optical luminosity together with the spin down power shows that PSR B1937+21 doesn't fall on a radio-active regime as shown in Figure 3.

It advisable to explore the spectral evolution and mass transfer of the pulsar by performing a direct observation and time series analysis. Comparison of the observation records and a new one will verify the correlation of the target parameters. Simulations and actual observation are recommended to test the evolution of pulsar as we analyse the optical properties of it.

My deepest gratitude comes first to my institute for funding my presentation. And to my research adviser, colleagues, and friends for sharing some insights. Especially, to my partner, thank you very much for understanding.

\section{References}

1. F. Ambrosino, A. Papitto, L. Stella, F. Meddi, P. Cretaro, L. Burderi, T. Di Salvo, G. L. Israel, A. Ghedina, L. Di Fabrizio, L. Riverol, Nature Astronomy (2018)

2. D.C. Backer, Astrophys. Astr. (1995)

3. G. Cusumano, T. Mineo, E. Massaro, et al. Astronomy and Astrophysics (2003)

4. S. Kisaka, and S. J. Tanaka, IOP Conf. Series: Journal of Physics: Conf. Series 932 (2017)

5. V.M. Kontorovich, Astronomy Reports volume 59, pages 277-287 (2015)

6. J. W. McKee, B. W. Stappers, C. G. Bassa, S. Chen, I. Cognard, M. Gaikwad, G. H. Janssen, R. Karuppusamy 2, M. Kramer, K. J. Lee, K. Liu, D. Perrodin, S. A. Sanidas, R. Smits, L. Wang, and W. W. Zhu, Nature Astronomy, 1-21 (2018)

7. L. Nicastro, G. Cusumano, O. L"ohmer, M. Kramer, L. Kuiper, W. Hermsen, T. Mineo, and W. Becker, Astronomy and Astrophysics (2004)

8. A. Patruno, S. Bogdanov, A. M. Archibald, C. Bassa, J. W. T. Hessels, G. H. Janssen, B. W. Stappers, arXiv: 1402.6324 [astro-ph.HE] Astrophysical Journal (2014)

9. B. W. Stappers, A. M. Archibald, J. W. T. Hessels, C. G. Bassa, S. Bogdanov, G. H. Janssen, V. M. Kaspi, A. G. Lyne, A. Patruno, S. Tendulkar, A. B. Hill, T. Glanzman, Nature Astronomy, (2014)

10. M. Takahashi, S. Shibata, K. Torii, Y. Saito, N. Kawai, M. Hirayama, T. Dotani, D. Gunji, H. Sakurai, I. H. Stairs, and R. N. Manchester, The Astrophysical Journal, 554:316-321 (2001) 